BMJ Open Sport \& Exercise Medicine

\title{
Osteochondral defects of the talus with a focus on platelet-rich plasma as a potential treatment option: a review
}

\author{
Ahmed Aly Elghawy, Carlos Sesin, Michael Rosselli
}

To cite: Elghawy AA, Sesin C, Rosselli M. Osteochondral defects of the talus with a focus on platelet-rich plasma as a potential treatment option: a review. BMJ Open Sport \& Exercise Medicine 2018;4:e000318. doi:10.1136/ bmjsem-2017-000318

Accepted 2 January 2018
Check for updates

Internal Medicine, Mount Sinai Medical Center, Miami Beach, Florida, USA

Correspondence to Dr Ahmed Aly Elghawy; ahelghawy@gmail.com

\section{ABSTRACT}

Objective To provide a review of osteochondral lesions of the talus, to discuss the evidence of the risks and benefits of platelet-rich plasma (PRP) as a viable treatment option, and to measure the efficacy of PRP using MRI evidence of cartilage regeneration, as well as scales that measure improvement in 'pain' and 'functionality'.

Eligibility criteria Studies that use PRP in either conservative or intraoperative settings to treat osteochondral defects of the talus.

Results There are seven studies that compare hyaluronic acid or standard surgical options against PRP in treating osteochondral lesions of the talus. Five studies use PRP as supplemental treatment in intraoperative settings, while two studies use PRP conservatively as intra-articular injections. There were minimal adverse effects. Pain and functionality scores consistently improved in those who underwent PRP treatments over the course of 4 years. MRI showed significant but inconsistent results in chondral regeneration. Conclusion PRP may show clinical benefit in those with osteochondral lesions of the talus in terms of pain and functionality, although chondral regeneration via MRI is inconsistent. Limitations include the small sample sizes in these seven studies, as well as no standardised formula for PRP preparation.

Clinical relevance To serve as an overview of the literature regarding PRP treatment for osteochondral lesions of the talus and how this modality may improve patient outcomes in pain, functionality and chondral regeneration. A case is reported to complement the subject review.

\section{CASE PRESENTATION}

A 25-year-old man with no significant medical history presents to the office for a complaint of right ankle pain, worsening gait and increasing difficulty ambulating for the last 14 months following a sports-related injury. He attributed his symptoms to an amateur wrestling match during which he felt a sudden pain in the lateral side of the right ankle after his body rolled over his ankle while the foot was in complete dorsiflexion and inversion. The pain was described as sharp, constant and non-radiating, localised to the lateral side of the right ankle, and was rated as an 'eight' on a scale of 1-10. The next morning, the site of injury was swollen, minimally improved with non-steroidal

\section{What is already known?}

Most osteochondral lesions of the talus are traumainduced and occur on the lateral side.

- The most cost-effective diagnostic modality is a precise history and physical; the most accurate diagnostic modality is direct visualisation via arthroscopy.

- Treatment is divided between conservative (nonsteroidal anti-inflammatory drugs, ankle brace and others) and surgical approaches (arthroscopy, debridement, transplant/grafting).

\section{What are the new findings?}

- Platelet-rich plasma (PRP) can be administered via intra-articular injections or via surgical approaches either alone or with an adjunct therapy (hyaluronic acid) or scaffold (collagen).

- PRP shows consistent improvement in pain relief and functionality, although MRI evidence of cartilage regeneration is variable.

- Limitations include few studies, lack of standard formula preparation and cost-effectiveness of treatment.

anti-inflammatory drugs (NSAIDs), and the patient required a cane for ambulating. The patient sought medical attention at a local outpatient clinic and had an X-ray of the right foot and ankle which demonstrated no fractures. The pain improved over the next few weeks with ice, mobility exercises and NSAIDs, but the patient noticed that his activities slowly decreased over the next 9 months. At first, the pain was only present during light outdoor jogs but over time had progressed to walking and only improved with rest. The quality of pain changed to a dull, 'gnawing' pain associated with reduction in range of motion in the ankle joint. The patient sought further medical therapy 7 months postinjury with intra-articular corticosteroids and weekly physical therapy, with minimal improvement. An MRI of the joint showed a 'cartilage defect with mild adjacent subchondral marrow 
edema in the far lateral aspect of the talar dome', as well as 'a small amount of tibiotalar joint fluid'. These findings were consistent with a 'talar-dome lesion' and signs of osteoarthritis. The patient was referred to a podiatrist for further recommendations based on the MRI findings.

Physical examination revealed a well-nourished man in minimal distress at rest, with vital signs within normal limits and stable. On focused examination, there was no pain in the right ankle at rest in a seated or supine position, although there was focal tenderness to palpation and pain with range of motion testing of the tibiotalar joint, specifically in dorsiflexion and inversion. Tenderness was also elicited with palpation just inferior to the lateral malleolus. His gait was antalgic, with noted compensatory body weight shift favouring on his left side.

The patient was offered surgical options versus 'platelet-rich plasma' (PRP) combined with synthetic hyaluronic acid (HA) injections in hopes of reducing pain and improving functional use of the right ankle. The patient chose intra-articular PRP injections, which were administered using an anterolateral and posterolateral approach.

\section{BACKGROUND}

The talus is the second largest tarsal bone in the ankle, articulates with the tibia, fibula, calcaneus and navicular, and is vital in weight transmission from the body to the foot, allowing for maintained balance during ambulation. It also assists in dorsiflexion and plantar flexion of the ankle, further aiding in movement of the foot with each step during ambulation. ${ }^{1}$ Although there are no muscles that originate or insert onto the talus, its connections to the adjacent bones via ligaments stabilise the ankle. ${ }^{2}$ Sixty per cent of the talar dome is lined with cartilage to allow for smooth, frictionless movement of the joint. The talus is supplied primarily by the dorsalis pedis, peroneal artery, posterior tibial artery and artery of the sinus tarsi. ${ }^{3}$

\section{AETIOLOGY/PATHOGENESIS}

Talar dome lesions, also known as osteochondral lesions of the talus, are injuries to the cartilage and underlying talus within the ankle joint. ${ }^{4}$ The two main types of injuries are ischaemic and traumatic, with the latter being the most common cause. In more than $85 \%$ of documented patients, a traumatic event is implicated in the development of the disease. ${ }^{56}$ Specifically, $98 \%$ of lateral lesions and $70 \%$ of medial lesions are associated with trauma based on Flick and Gould's ${ }^{7}$ review of more than 500 documented talar dome lesions. These incidents can be a singular, acute macrotraumatic incident, or a repetitive, cumulative microtrauma-induced event. There is a slight male predominance in incidence of injury, presenting most commonly in those between the ages of 20 and 30 years. Up to $6.5 \%$ of all ankle sprains lead to osteochondral lesions, and about $10 \%$ of these lesions present bilaterally. ${ }^{3}$ The inverse relationship between cartilage thickness and mean compressive modulus has also helped further correlate aetiology to site of injury. Tangential, shallower injuries to the lateral side indicate more acute events, while the stiffer tibial cartilage on the medial side requires more repetitive trauma in a perpendicular force vector to elicit injury. ${ }^{8}$

To a lesser degree, osteochondral lesions occur in association with ischaemic necrosis when there is interference of the blood supply to a given portion of the talus. This is often labelled as 'Osteochondritis Dissecans' (OCD), where subchondral bone ischaemia can further evolve to separation of the bone and cartilage. This occurs in a non-traumatic fashion, commonly identified in children and adolescents, and can lead to skeletal immaturity due to vascular impairment. With lack of resources to encourage neoangiogenesis, the development of avascular necrosis (AVN) becomes more likely. Patients with these lesions typically do not have a previous macrotraumatic event as the inciting factor, and thus occur more commonly on the medial side. The presence of intact cartilage over an area of subchondral necrosis can be a good prognostic sign of potential for spontaneous healing, whereas absence of cartilage has a less favourable likelihood of improvement without intervention. It has been proposed that adolescents with complete cartilage OCD may have genetic risk factors that predispose them to developing the disease, although as per Zanon $e t$ $a \ell^{\natural}$ in 'Osteochondritis Dissecans of the Talus', 'there are no ongoing studies to identify such genes'.

\section{CLINICAL PRESENTATION}

Ankle pain may initially present acutely following an injury or insidiously in those whose lives and professions require frequent and prolonged ambulation. The pain may be localised to the medial or, more commonly, the lateral side, is described as dull or achy, is exacerbated with exertion and weightbearing, and relieved temporarily with rest and overthe-counter (OTC) pain medications, such as NSAIDs. Pain typically may develop acutely following an ankle inversion and in sports that involve abrupt stops and changes in direction, repetitive high-impact actions to the lower extremities, and physical contact, that is, tackling. Younger, active patients tend to describe an inciting event with acutely worsening pain when attempting to reproduce the event, while older patients endorse a slow progression of mild pain and inability to perform their normal activities of daily living. ${ }^{10}$ In addition, patients may complain of ankle locking, clicking or 'giving out', and gait instability with prolonged use. ${ }^{3}$ Patients may delay seeking medical attention while attempting to self-treat with rest and OTC pain-relieving medications. Failure to manage symptoms conservatively can lead to either stagnant or worsening symptoms by the time they are seen by a medical professional. Complications include post-traumatic arthritis secondary to cartilage damage, and AVN or osteonecrosis with collapse secondary to bone death from disrupted blood supply. ${ }^{11}$

\section{DIAGNOSIS}

The history and physical exam are an invaluable first step in evaluating talar dome lesions. Clinical suspicion can be further strengthened with a thorough physical 


\begin{tabular}{|c|c|}
\hline Stage & Description \\
\hline 1 & Subchondral compression fracture \\
\hline Ila & Chondral fracture (partial avulsion) \\
\hline $\mathrm{llb}$ & Subchondral cyst \\
\hline III & $\begin{array}{l}\text { Chondral fracture with separated segment (non- } \\
\text { displaced) }\end{array}$ \\
\hline IV & $\begin{array}{l}\text { Chondral fracture with separated segment } \\
\text { (displaced) }\end{array}$ \\
\hline
\end{tabular}

exam revealing joint swelling, crepitus, reduced passive and active range of motion, and tenderness to palpation (especially with lateral lesions). Patients may also complain of pain with gait assessment, often favouring the contralateral foot for weightbearing. Depending on the chronicity of the complaint, patients may also endorse contralateral knee, hip and back pain due to compensatory mechanisms. Unfortunately, there are no specific physical exam tests that confirm the diagnosis, although assessing for laxity with anterior drawer and talar tilt to observe the anterior talofibular ligament and calcaneofibular ligament, respectively, can aid in narrowing the diagnosis. Failure of conservative management may necessitate imaging studies to further visualise the anatomy and confirm the diagnosis. ${ }^{3}$

\section{IMAGING}

Initial imaging following an acute ankle injury involves plain radiographs of the joint, preferably weightbearing if tolerable in three views (anteroposterior (AP), lateral and mortise views). ${ }^{12}$ Lateral lesions are best viewed in the mortise view, demonstrating a 'shallow, wafer-shaped lesion', while medial lesions are ideally shown in the AP view demonstrating a deep, cup-shaped lesion. ${ }^{13}$ Bilateral radiographs should be obtained for comparison, and it should be noted that the incidence of a contralateral lesion is anywhere between $10 \%$ and $25 \% .{ }^{12}$ Radiographic classification and staging by Berndt and Harty in 1959 are listed in table $1 .{ }^{14}$ While CT in the coronal and axial views can improve visualisation of bone in comparison with plain radiographs, it has poor visualisation of cartilaginous surfaces. ${ }^{15}$ In 1996, Ferkal modified the previous classification system of Berndt and Harty with findings based on CT, listed in table $2 .{ }^{16}$ Instead, MRI is

\begin{tabular}{ll}
\hline Table 2 & Ferkel classification $(\mathrm{CT})^{32}$ \\
\hline Stage & Description \\
\hline I & $\begin{array}{l}\text { Cystic lesion with dome of talus (intact roof) } \\
\text { Ila }\end{array}$ \\
$\begin{array}{l}\text { Cystic lesion with communication to talar dome } \\
\text { surface }\end{array}$ \\
III & $\begin{array}{l}\text { Open articular surface lesion with overlying } \\
\text { undisplaced fragment }\end{array}$ \\
III & $\begin{array}{l}\text { Undisplaced lesion with lucency } \\
\text { IV }\end{array}$ \\
\hline
\end{tabular}

\begin{tabular}{|c|c|}
\hline Stage & Description \\
\hline । & Articular cartilage injury \\
\hline Ila & $\begin{array}{l}\text { Cartilage injury with bony fracture and oedema } \\
\text { (acute) }\end{array}$ \\
\hline $\mathrm{llb}$ & $\begin{array}{l}\text { Cartilage injury with bony fracture and oedema } \\
\text { (chronic) }\end{array}$ \\
\hline III & $\begin{array}{l}\text { Detached, non-displaced bony fragment with } \\
\text { fluid rim beneath fragment }\end{array}$ \\
\hline IV & $\begin{array}{l}\text { Displaced fragment, uncovered subchondral } \\
\text { bone }\end{array}$ \\
\hline V & Subchondral cyst present \\
\hline
\end{tabular}

considered the gold standard in recognising osteochondral lesions, with $100 \%$ specificity and anywhere between $73 \%$ and $95 \%$ sensitivity. ${ }^{15}$

Less sensitivity, likely secondary to the thickness of imaging slices on MRI ( $3 \mathrm{~mm}$ taken in $1 \mathrm{~mm}$ intervals), compared with the thickness of the talar cartilage $(0.4-2.1 \mathrm{~mm})$, means that the false-negatives are possible, so a negative MRI with clinical symptoms requires further work-up with arthroscopy ${ }^{16}$ Further staging based on MRI was developed by Hepple $e t$ al, listed in table $3{ }^{17}$

\section{ARTHROSCOPY}

The most definitive way to diagnose an osteochondral talar dome lesion is via direct visualisation from arthroscopy. This method is used when the patient has negative imaging studies but is still clinically symptomatic despite conservative management. A small incision is made in the skin and an arthroscope with a lens is inserted. This allows for visualisation of the cartilage, bone and ligaments with immediate intervention if needed. In this sense, this modality is the only one that is both diagnostic and therapeutic. The Ferkel/Cheng classification based on arthroscopic findings are listed in table $4 .{ }^{17}$

\section{MANAGEMENT}

Treatment of osteochondritic talar dome lesions depends on the locations of the lesion, the severity of the injury as well as the stability of the cartilage. Non-surgical approaches are considered initial therapy in stages I and II lateral lesions and stages I, II and III medial lesions.

\begin{tabular}{ll}
\hline $\begin{array}{l}\text { Table } 4 \\
\text { Findings) }\end{array}$ & Ferkel classification ${ }^{34}$ (Arthroscopic Cartilage \\
\hline Grade & Description \\
\hline A & Smooth, intact but soft \\
B & Rough surface \\
C & Fibrillations and fissures \\
D & Flap present or bone exposed \\
E & Loose undisplaced fragment \\
F & Displaced fracture \\
\hline
\end{tabular}


Stable lesions, or lesions where there are no loose pieces of cartilage or bone in the joint, can also first be approached via non-surgical means. Immobilisation of the joint, by way of a cast or immobilising boot for 6-12 weeks, can help protect the talus from further injury. As the patient tolerates more weightbearing activity, the cast can be switched to an ankle brace to stabilise the joint and prevent reinjury. NSAIDs can reduce inflammation and pain and may allow the patient to tolerate physical therapy exercises in hopes of improving range of motion and strength. Depending on the severity of the lesion, expected recovery time may be from weeks to months, and possibly longer with reinjury. Unfortunately, outcomes for non-operative treatments are unsatisfactory, where as many as $50 \%$ of patients do not reach complete, asymptomatic recovery. ${ }^{4} 18$

In lesions that are refractory to non-surgical treatments, or lesions that are unstable, surgery should be considered. The goal is to restore the normal shape and surface of the talus in order to mimic the normal motion and joint forces that are needed for proper use with nominal symptoms. As noted before, arthroscopic instruments may be used to visualise and remove fragments in a minimally invasive manner. In larger injuries or injuries with less accessible lesions, an open approach may be necessary. Surgical treatments include debridement, open reduction internal fixation (ORIF) of the fragment, microfracture/drilling of the lesion, and/or transplant/ grafting of bone and cartilage. Preoperative imaging is necessary to characterise the lesion, and depending on the size and location the type of surgery and the site of entry will differ. ${ }^{618}$ Outcomes are favourable, where more than $70 \%$ of patients show improved outcomes from debridement and drilling. As with any surgical procedure, there are risks and complications that warrant discussion, which include but are not limited to infection, phlebitis, non-union, damage to adjacent tendons and neurovascular anatomy, worsening pain, swelling, bleeding, instrumentation breakage, and need for repeat surgeries (especially in the case of transplantations). These postsurgical complications are rare with an incidence of less than $1 \%$ of all arthroscopic procedures. ${ }^{19}$ Recovery time in these patients tends to be longer than non-surgical approaches, with pain commonly seen in patients for up to 1 year, and for regular repeat imaging to be performed via MRI or CT. ${ }^{18}$

In patients who fail non-surgical therapy but are hesitant to undergo surgery, less invasive modalities, such as intra-articular HA or PRP injections, may be considered. HA has been used in patients with osteoarthritis as an injectable therapy for 'restoring the viscoelastic properties of synovial fluid, which is reduced in OA (Osteoarthritis) $.1{ }^{14}$ As for PRP, although there is theoretical potential in self-repair via growth factor induction, the use of PRP is a widely controversial and debated treatment option that warrants discussion.

\section{PLATELET-RICH PLASMA}

PRP, by definition, is a concentration of platelets that are extracted from autologous blood. Platelets are 'small, nonnucleated bodies, produced by megakaryocytes in the bone marrow, that circulate in peripheral blood and are known primarily for their role in hemostasis'. ${ }^{20}$ These bodies harbour a number of cytokines that induce cell proliferation, differentiation, and most importantly wound healing. These cytokines include transforming growth factor-beta (TGF- $\beta$ ), platelet-derived growth factor, insulin-like growth factor (IGF-I and IGF-II), fibroblast growth factor, epidermal growth factor, vascular endothelial growth factor and endothelial cell growth factor. ${ }^{21}$ With over 300 molecules signalled by platelet induction, there could be a theoretical benefit in tissue repair with platelet activation at the site of injury. TGF- $\beta$ is considered to be the most active participant in anabolic chondrocyte production and increased bone formation. In addition to stimulating the migration of bone-marrow stromal cells towards the lesion in question, TGF- $\beta$ can further aid in natural recovery by directing chondrocyte differentiation and sustaining chondrocyte phenotype. ${ }^{22}$

To prepare PRP, peripheral blood is drawn manually and concentrated via centrifugation. This separation of blood products enables platelets to degranulate and release the aforementioned growth factors, allowing for easy extraction of the activated platelet products. PRP, once isolated, can be injected directly (with or without ultrasound-guided aid) into the lesion site or may be delivered in conjunction with a scaffold (such as collagen) or with adjunct therapy, such as HA or a synthetic HA substitute. ${ }^{22}$

Most evidence regarding the efficacy of PRP on the ankle is extrapolated from results obtained from PRP application of the knee joint. While promising results are noted in knees when compared with HA or saline solution, studies regarding PRP on the ankle are lacking. At this time, there have been seven published papers discussing the use of PRP on osteochondritic lesions of the ankle, of which five involve augmenting surgical repair of the cartilage, and two which discuss intra-articular, or conservative, injections. ${ }^{23}{ }^{24}$ In a randomised controlled study by Mei-Dan et al, 30 patients with talar dome lesions refractory to conservative management were split into two groups. Half the patients were given intra-articular PRP while the other was given intra-articular HA, with three weekly injections per group. The patients were followed and re-evaluated at 28 weeks to assess for pain, stiffness and function using the Ankle Hindfoot Score (AHFS), the American Orthopaedic Foot and Ankle Score (AOFAS) and the Visual Analogue Scale (VAS). At 28 weeks, the patients who underwent PRP injections had significantly less pain and improved function. ${ }^{25}$ In Angthong et als study, a considerably weaker analysis of only five patients with osteoarthritis were given PRP under ultrasound or fluoroscope and evaluated with the VAS Foot and Ankle Score, showing significant clinical improvement at 16 months. These patients were also evaluated radiographically via MRI, although no anatomical improvement was visualised 
at 5 months postinjection. ${ }^{26}$ Of note, there were no adverse effects from the PRP injections reported in these two studies.

In Guney et als randomised comparative study, 35 patients with full-thickness talar dome lesions were scheduled to undergo arthroscopic microfracture alone or combined with PRP (administered 6-24hours after microfracture). Although patients who underwent the combined treatment had higher VAS for pain at 12-24 months postsurgery, the overall functional score via AOFAS and Foot and Ankle Ability Measure improved significantly in that same time. ${ }^{27}$

In a study by Giannini et al, bone marrow-derived cells were harvested from the posterior iliac crest and combined with mesenchymal stem cells and PRP and then scaffolded with either collagen or HA. This composite was then placed at the lesion site via arthroscopy, and patients were followed up at $6,12,18$ and 24 months, with significant improvement in AOFAS score at 6 months and up to $75 \%$ of patients returning to 'high impact sport activity' at 11 months. Five patients were taken back for second looks via arthroscopies, and in two cases biopsies were performed showing new cartilage formation. ${ }^{17}$ At 4 years, the AOFAS score was still significantly improved compared with baseline, although optimal scores were recorded at 24 months, with significant decreases at 36 and 48 months. Despite these results, MRI findings at 48 months showed hyaline-like regenerated cartilage in greater than $80 \%$ of the treated lesion. ${ }^{28}$

In a follow-up study by the same authors, 81 patients were split into three groups: open autologous chondrocyte implantation (ACI), arthroscopic ACI and bone-marrow-derived mesenchymal cells 'one-step' technique. All three groups had significant clinical improvement via AOFAS, and there was no significant difference between groups. Radiographically, MRI showed similar improvement in cartilage defect filling between groups. ${ }^{29}$ Battaglia $e t a l^{30}$ further analysed the one-step technique by evaluating the MRIs on 20 patients at 2 years postoperatively, showing $78 \%$ hyaline cartilage repair of the lesion. Economically, the 'one-step' approach costs less than half of the traditional arthroscopic ACI, showing promise for widespread use in the future. ${ }^{29}$

As convincing as this evidence appears in these seven studies, there are several limitations that still need to be addressed. The biggest issue comes in the formulation of PRP and the lack of a universal standard of preparation, making the results in these studies difficult to apply broadly in clinical practice. Without a standard protocol for PRP preparation or administration, access to PRP becomes difficult since insurance often will not cover treatment elective modalities that do not definitively supplant standard of care modalities. Additionally, the numerous molecules involved in activation and bioregeneration pose the issue of determining exact mechanisms of healing. Without directly comparing these studies with solo PRP therapy, it is difficult to attribute all the measured benefits directly to PRP instead of the combined therapies mentioned
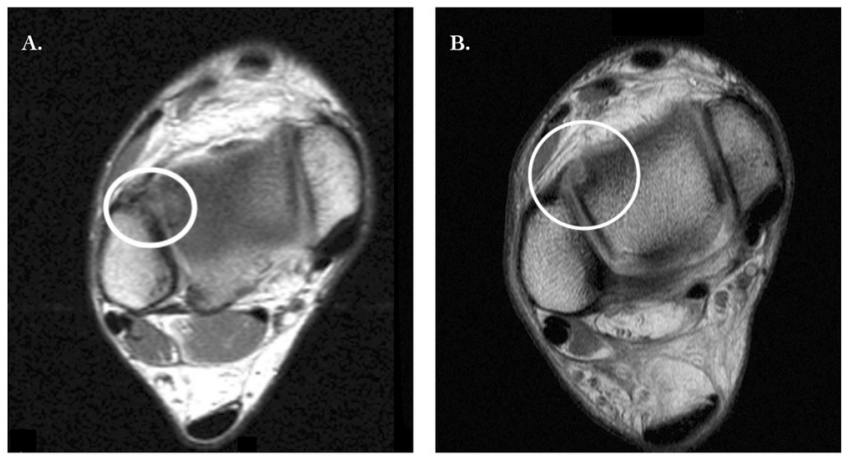

Figure $1 \mathrm{MRI}$ of the right ankle (axial views) at 4 months prior to platelet-rich plasma (PRP) intra-articular injection (A) and 36 months post PRP intra-articular injection (B).

in the studies above. And although promising, the few studies presented have small sample sizes, have shortterm follow-up and have not been measured beyond 48 months, meaning more research over a longer period of time is needed before definitive statements on the efficacy of PRP can be made. ${ }^{2024}$

Future studies comparing solo PRP therapy against PRP with adjunct HA, HA alone, surgery and a control (perhaps conservative NSAID therapy, physical therapy or both) could be interesting and help elucidate the role of PRP in managing patients with osteochondral lesions of the talus.

\section{BACK TO THE CASE}

Following the initial round of intra-articular PRP injections (with HA scaffolding), the patient was assessed on a biweekly basis for 3 months via range of motion testing, gait analysis and pain assessment. The patient was reassessed at 1 year, and at that time the patient had no pain or restriction and had full functionality per the Ankle Hindfoot Scale and the AOFAS. Repeat MRI at 36 months post intra-articular PRP injections demonstrated '...cartilage erosion and subchondral cyst anterolaterally in the talar dome'. There was no evidence of regenerative improvement per MRI despite clinical resolution of pain and no restrictions in physical activity. Figures 1 and
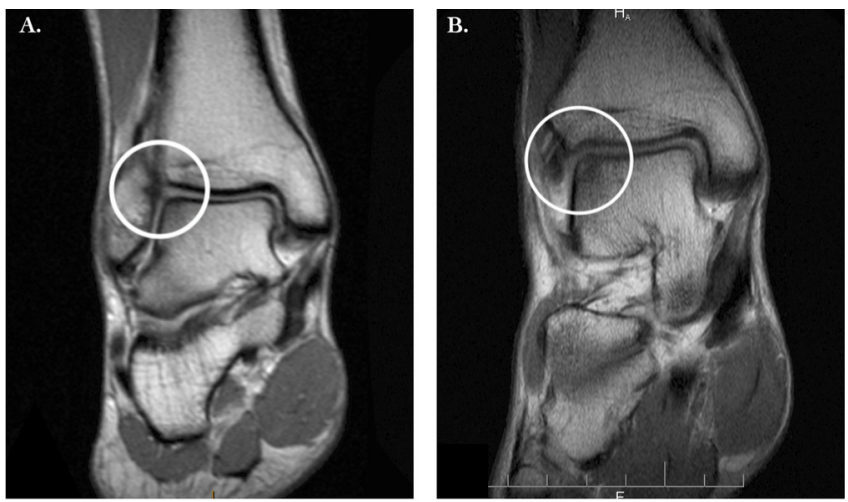

Figure $2 \mathrm{MRI}$ of the right ankle (coronal views) at 4 months prior to platelet-rich plasma (PRP) intra-articular injection (A) and 36 months post PRP intra-articular injection (B). 
2 compare the the right ankle on MRI before and after intra-articular injections.

\section{SUMMARY AND RECOMMENDATIONS}

Osteochondral lesions of the talus, or talar dome lesions, can be classified as ischaemic or traumatic injuries and can be debilitating if untreated. Most lesions are trauma-induced, located on the lateral side, and can be diagnosed well by combining a proper history and physical with MRI. Arthroscopy is the most definitive diagnostic modality as it allows for direct visualisation and is the only diagnostic modality that is also therapeutic. Treatment modalities include non-surgical management via physical therapy and anti-inflammatory medications, and surgical methods via open or arthroscopic debridement, ORIF, microfracture and grafting. PRP, a relatively newer modality that promotes chondral healing via platelet activation and biomarker induction, is a treatment that can be administered as an alternative to or in conjunction with surgical approaches. While studies so far may show improved clinical outcomes in pain and functionality of the joint with minimal adverse effects, there are no long-term data or universal PRP preparation model to definitively promote this treatment as standard of care. Even so, the future of PRP in the treatment of osteochondral lesions of the talus is promising.

Acknowledgements We would like to thank the patient, who wished to remain anonymous, for allowing his case to be written and presented with his express permission.

Contributors AAE contributed the idea of the manuscript, initial registration of review, initial search, review of data, initial drafting and editing of manuscript. CS and MR contributed to editing of the manuscript. All authors approved the final draft of the manuscript.

Funding None declared.

Competing interests None declared.

Ethics approval All previous studies included in this review have been approved by the ethics committees before their publication.

Provenance and peer review Not commissioned; externally peer reviewed.

Data sharing statement Data available on request.

Open Access This is an Open Access article distributed in accordance with the Creative Commons Attribution Non Commercial (CC BY-NC 4.0) license, which permits others to distribute, remix, adapt, build upon this work non-commercially, and license their derivative works on different terms, provided the original work is properly cited and the use is non-commercial. See: http://creativecommons.org/ licenses/by-nc/4.0/

(C) Article author(s) (or their employer(s) unless otherwise stated in the text of the article) 2018. All rights reserved. No commercial use is permitted unless otherwise expressly granted.

\section{REFERENCES}

1. "Talus Bone Pictures, Function \& Diagram | Body Maps". Healthline: Healthline Media. 16 June 2017.

2. "Talus". Kenhub. N.p. 16 June 2017.

3. Trousdale RT, Robert T, Diane L, et al. "Osteochondral Lesions of the Talar Dome. " Orthopaedic Surgery Board Review Manual 13.2, 1981:176.

4. "Talar Dome Lesion". Foot Health Facts. N.p, 2017. 16 June 2017.

5. Anderson IF, Crichton KJ, Grattan-smith T, et al. Osteochondral Fractures of the Dome of the Talus." The Journal of Bone \& Joint Surgery. The Journal of Bone \& Joint Surgery 2017;71.

6. "Osteochondral Lesions of the Talus". Background, Anatomy, Pathophysiology. N.p, 2017. 16 June 2017.
7. Flick AB, Gould N. "Osteochondritis Dissecans of the Talus (transchondral Fractures of the Talus): Review of the Literature and New Surgical Approach for Medial Dome Lesions.". Foot \& Ankle: U.S. National Library of Medicine, 2017. 16 June 2017.

8. Shepherd DET, Seedhom BB. Thickness of Human Articular Cartilage in Joints of the Lower Limb". Annals of the Rheumatic Diseases: BMJ Publishing Group Ltd. 16 June 2017.

9. Zanon G, DI Vico G, Marullo M. Osteochondritis dissecans of the talus. Joints 2014;2:115-23.

10. Vannini F, Costa GG, Caravelli S, et al. Treatment of osteochondral lesions of the talus in athletes: what is the evidence? Joints 2016;4:111-20.

11. "Talus Fractures". Ortholnfo - AAOS. N.p, 2014. 16 June 2017.

12. Stone JW. Osteochondral Lesions of the Talar Dome. J Am Acad Orthop Surg 1996:4:63-73.

13. Judd D, Kim D. "Foot Fractures That Are Frequently Misdiagnosed As Ankle Sprains". American Family Physician. N.p. 16 June 2017.

14. Battaglia M, Guaraldi F, Vannini F, et al. Efficacy of ultrasound-guided intra-articular injections of platelet-rich plasma versus hyaluronic acid for hip osteoarthritis. Orthopedics 2013;36:e1501-e1508.

15. Joshy S, Abdulkadir U, Chaganti S, et al. Accuracy of MRI scan in the diagnosis of ligamentous and chondral pathology in the ankle. Foot Ankle Surg 2010;16:78-80.

16. Rolf CG, Barclay C, Riyami M, et al. The importance of early arthroscopy in athletes with painful cartilage lesions of the ankle: a prospective study of 61 consecutive cases. J Orthop Surg Res 2006;1:4.

17. Giannini S, Buda R, Vannini F, et al. One-step bone marrow-derived cell transplantation in talar osteochondral lesions. Clin Orthop Relat Res 2009;467:3307-20.

18. Osteochondral Lesion. Osteochondral Lesion: The American Orthopaedic Foot \& Ankle Society (AOFAS), 16 June 2017.

19. "Arthroscopy". Ortholnfo - AAOS. Ortholnfo - AAOS.org.

20. Foster TE, Puskas BL, Mandelbaum BR, et al. Platelet-Rich Plasma: From Basic Science to Clinical Application. Am J Sports Med 2017;37:2259-72.

21. Sun $Y$, Feng $Y$, Zhang $C Q$, et al. The regenerative effect of plateletrich plasma on healing in large osteochondral defects. Int Orthop 2010;34.

22. Zhu Y, Yuan M, Meng HY, et al. Basic science and clinical application of platelet-rich plasma for cartilage defects and osteoarthritis: a review. Osteoarthritis Cartilage 2013;21:1627-37.

23. Mei-Dan O, Carmont MR, Laver L, et al. Platelet-Rich Plasma or Hyaluronate in the Management of Osteochondral Lesions of the Talus. Am J Sports Med 2012;40:534-41.

24. Vannini F, Di Matteo B, Filardo G. Platelet-rich plasma to treat ankle cartilage pathology - from translational potential to clinical evidence: a systematic review. J Exp Orthop 2015;2:2:2.

25. Khoshbin A, Leroux T, Wasserstein D, et al. The efficacy of plateletrich plasma in the treatment of symptomatic knee osteoarthritis: a systematic review with quantitative synthesis. Arthroscopy 2013;29:2037-48.

26. Angthong $\mathrm{C}$, Khadsongkram A, Angthong W. Outcomes and quality of life after platelet-rich plasma therapy in patients with recalcitrant hindfoot and ankle diseases: a preliminary report of 12 patients. $J$ Foot Ankle Surg 2013;52:475-80.

27. Guney A, Akar M, Karaman I, et al. "Clinical Outcomes of Platelet Rich Plasma (PRP) as an Adjunct to Microfracture Surgery in Osteochondral Lesions of the Talus." Knee Surgery, Sports Traumatology. Arthroscopy 2013;23.8:2384-9.

28. Giannini S, Buda R, Battaglia M, et al. One-Step Repair in Talar Osteochondral Lesions. Am J Sports Med 2013;41:511-8.

29. Giannini S, Buda R, Cavallo M, et al. Cartilage repair evolution in post-traumatic osteochondral lesions of the talus: From open field autologous chondrocyte to bone-marrow-derived cells transplantation. Injury 2010;41:1196-203.

30. Battaglia M, Rimondi E, Monti C, et al. Validity of T2 mapping in characterization of the regeneration tissue by bone marrow derived cell transplantation in osteochondral lesions of the ankle. Eur $J$ Radiol 2011;80:e132-e139.

31. BERNDT AL, HARTY M. Transchondral fractures (osteochondritis dissecans) of the talus. J Bone Joint Surg Am 1959;41-A:988-1020.

32. Ferkel RD, Zanotti RM, Komenda GA, et al. Arthroscopic treatment of chronic osteochondral lesions of the talus: long-term results. Am J Sports Med 2008;36:1750-62.

33. Hepple S, Winson IG, Glew D. Osteochondral lesions of the talus: a revised classification. Foot Ankle Int 1999;20:789-93.

34. Ferkel RD, Cheng MS, Applegate GR, 1995. A new method of radiologic and arthroscopic staging for osteochondral lesions of the talus [abstract]. Proceedings of the American Academy of Orthopaedic Surgeons 62nd Annual Meeting, Orlando, Florida 16-21 